\title{
Locations of Bromide Ions in Tetragonal Lysozyme Crystals
}

\author{
Kap Lim," Arunan Nadarajah," Elizabeth L. Forsythe." and Marc L. Pusey ${ }^{\prime *}$ \\ "Department of Biochemistry and Biophysics, Texas A\&M University. College Station, TX 77843, USA, "Department \\ of Chemical and Environmental Engineering, University of Toledo, Toledo, OH 43606, USA, "Universities Space \\ Research Association, 4950 Corporate Drive, Suite 100, Huntsville, AL. 35806, USA, and 'Biophysics Branch ES76, \\ NASA/Marshall Space Flight Center, Huntsville, AL 35812, USA. E-mail: pusey@crystal.msfc.nasa.gov
}

(Received 27 August 1997; accepted lo February 1998)

\begin{abstract}
Anions have been shown to play a dominant role in the crystallization of chicken egg-white lysozyme from salt solutions. Previous studies employing X-ray crystallography have found one chloride ion binding site in the tetragonal crystal form of the protein and four nitrate ion binding sites in the monoclinic form. In this study the anion positions in the tetragonal form were determined from the difference Fourier map obtained from lysozyme crystals grown in bromide and chloride solutions. Five possible anion-binding sites were found in this manner. Some of these sites were in pockets containing basic residues while others were near neutral, but polar, residues. The sole chloride ion binding site found in previous studies was confirmed, while four further sites were found which corresponded to the four binding sites found for nitrate ions in monoclinic crystals. The study suggests that most of the anionbinding sites in lysozyme remain unchanged even when different anions and different crystal forms of lysozyme are employed.
\end{abstract}

\section{Introduction}

Most proteins bind with counterions in solution. When these proteins are crystallized, many of these ions may be expected to remain bound to the protein. The tetragonal form of chicken egg-white lysozyme (CEWL), is usually crystallized at a pH between 4 and 5 , which is well below its isoionic point of 11.4. The protein has positive net charge in this $\mathrm{pH}$ range (Tanford \& Wagner, 1954), mostly interacting with, or binding to. anions in solution. Thus, anions may be expected to predominate among the bound counterions in crystals of this protein.

The importance of anions in the crystallization of lysozyme is known from their effect on the crystal solubility and the crystal form. Early investigations showed that tetragonal crystals were produced when chloride and bromide salts were employed as precipitants, whilst iodide and thiocyanate salts produced monoclinic crystals and nitrate salts gave monoclinic or triclinic crystals (Alderton \& Fevold, 1946: Steinrauf,
1959: King. 1959). Crystal solubilitics showed a similar trend with thiocyanate ions causing the crystals to be least soluble and chloride ions causing them to be more soluble (Riès-Kautt \& Ducruix, 1989; Pusey \& Munson, 1991). Recently, the locations of hound nitrate ions in monoclinic lysozyme crystals have been reported (Rao \& Sundaralingam. 1996). Knowing the number and locations of anions bound to the protein in the different crystal forms may lead to an understanding of the anioninduced polymorphism of lysozyme crystals, and help our understanding of the protein-crystallization process itself.

Earlier studies of tetragonal CEWL have located some of the binding sites. In their pionecring study of the structure of lysozyme in the tetragonal form. Phillips and co-workers found only one bound chloride ion (Blake et al., 1967). This was localed between two molecules related by $4_{3}$ symmetry. Binding studies have also been performed with phenolsulfothalein dyes in order to understand the enzymatic activity of lysozyme (Krishnamoorthy \& Prabhananda, 1982a,b). A crystallographic study revealed two large pockets on the protein surface near the active-site cleft to be the binding sites for these dyes (Madhusudan \& Vijayan, 1992). In other physico-chemical (i.e. non-crystallographic) binding studies the number of anions found includes those present in the solvent channels, making it impossible to determine those rigidly bound only to the crystalline matrix (Palmer et al., 1948; Carr, 1953; King, 1959). However, these studies do suggest that there may be more anion-binding sites in tetragonal lysozyme than the single site found by Phillips and co-workers. Very recently, in a high-resolution crystallographic study, the presence of an intermolecular anion-binding site was confirmed along with a sodium ion-binding site (Vaney et al., 1996).

In this study we investigated the binding sites of chloride and bromide ions in tetragonal lysozyme crystals employing ion-substitution crystallization experiments. The crystals were analyzed by X-ray crystallography, with the anions located from peaks in the electron-density difference map. These locations werc also compared with the anion-binding sites of lysozyme located in earlier studies.

Acta Crvatallographia Section $D$ ISSN $0907.4449 ; 1998$ 
Table 1. Summary of least-squares refinement parameters

Reflections from 6.0 to $1.8 \AA$ with $I>\sigma(l) \quad 1107.5$

Crystallographic $R$ factort

$R_{\text {iric factor }} \quad 0.240$

Percent reflections used to compute $R_{\text {fit's }}$

Number of protein non-H atoms

Number of ordered water molecules $\quad 85$

Number of bromide ions

R.m.s. deviation of bond distances (A)

R.m.s. deviation of bond angle ( )

R.m.s. deviation of dihedral angle ( )

R.m.s. deviation of improper angle ( )

Mcan $B$ fictor $\left(\mathrm{A}^{-}\right)$

Main-chain atoms

Side-chain atoms

Water molecules

Bromide ions

Ramachandran plot quality (\%)

Most-favored

Additional allowed

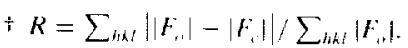

\section{Materials and methods}

\subsection{Protein preparation and crvstallization}

CEWL purchased from Sigma was repurified as previously described (Ewing et al., 1996). Tetragonal crystals were grown in sitting drops at $\mathrm{pH} 4.6$ in $0.1 \mathrm{M}$ sodium acetate buffer. Lysozyme chloride crystals were grown from solutions having a final concentration of

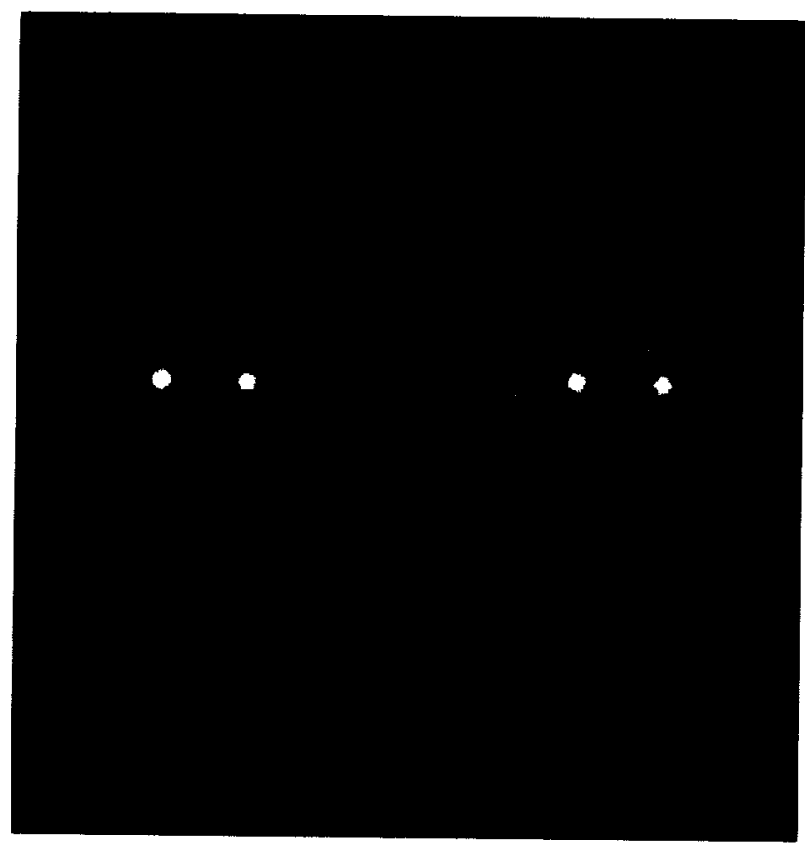

Fig. 1. The Hall anions (represented by solid spheres) at molecular interfaces in the tetragonal lysozyme crystal as viewed on the ab plane. These anions hold together the molecules forming the 4 helices. The square represents the unit cell. The anions and protein molecules are made different colours only to assist in distinguishing them.
$30 \mathrm{mg} \mathrm{ml}^{-1}$ protein and $5 \% \mathrm{NaCl}$, while the bromide crystals wele grown from $85 \mathrm{mg} \mathrm{ml}^{-1}$ protein and $0.5 \mathrm{M}$ $\mathrm{NaBr}$ solut ons. All crystals were grown at $293 \mathrm{~K}$.

\subsection{X-ray diffraction data collection and structure refinement}

$X$-ray defraction data were collected at room temperature, $\sim 297 \mathrm{~K}$, with a Rigaku R-AXIS II imaging-place diffractometer mounted on a Rigaku RU200 X-rity generator and processed with DENZO and SCALIPACK programs. Reflections for lysozyme bromide crystals were collected to $1.8 \AA$ resolution [98.2\% complete, $\left.I / \sigma(I)=8.1, R_{\text {merge }}=5.3 \%\right]$. Those for lysozyme culoride crystals were collected to $1.7 \AA$ $\left[97.5 \%\right.$ complete, $\left.I / \sigma(I)=6.6, R_{\text {merge }}=6.4 \%\right]$

Structura refinements were performed for the lysozyme brom de data, while the lysozyme chloride data were only used to compute a difference Fourier map to identify bronide-ion positions. Refinements began with the coordit ates of entry 6LYZ (Diamond, 1974), obtained frcm the Brookhaven Protein Data Bank. The space grosp $P 4_{32} 2$ and unit-cell parameters $a=b=79.0 . \AA$ and $c=38.14 \AA$ were used. A molecularreplacement search confirmed the position of the lysozyme molecule. Initially, simulated annealing (SA) calculations were performed with the program $X$-PLOR (Brünger, 1491). These refinements used the reflection data in the $(.0-2.7 \AA$ range, with a starting temperature of $3000 \mathrm{~K}$ which was gradually lowered to $300 \mathrm{~K}$.

Positional and temperature-factor refinements continued with the 6.0-2.7 $\AA$ reflections and included higher resolution data in each successive cycle. The difference Furier map $F_{r}(\mathrm{Br})-F_{c}(\mathrm{Cl})$ was computed at $2.3 \AA$ resolu ion to identify bromide-ion positions. The refinement continued for the lysozyme bromide model with the adcition of ordered water molecules from the $2.3 \AA$ resolution data, which were assigned when $F_{o}-F_{\text {. }}$ peaks greater than $3 \sigma$ were within hydrogen-bond distance $(<.5 \AA)$ of the lysozyme atoms and other previously ditermined solvent atoms. The final refinements included the $6.0-1.8 \AA$ reflections. $\neq$

\subsection{Comparsons with other lysozyme binding sites}

The variots hen egg-white lysozyme structures in the Protein Datı Bank were analyzed for any reported anion locaticns and were visualized using the program QUANTA (Folccular Simulations Inc.). In particular, the anion-bilding sites in lysozyme reported in four recent crysallographic studies were considered (Madhusudalı \& Vijayan, 1992; Madhusudan et al., 1993;

t Atomic coord nates and structure factors have besn deposited with the Protein Dat: Bank. Brookhaven National lahoratory (Reference: IA7.F). 

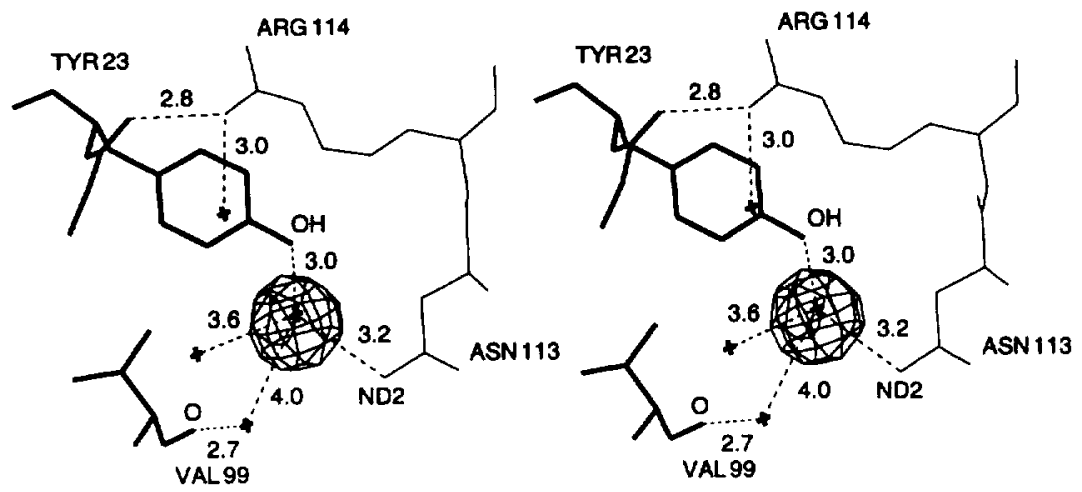

(a)
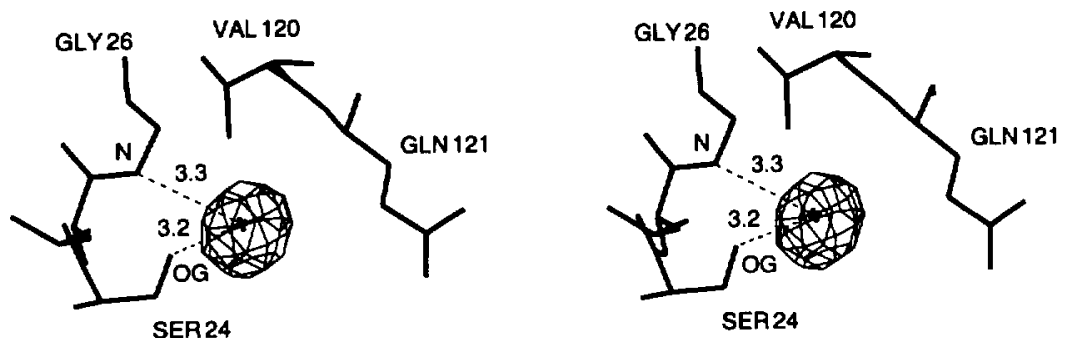

(b)
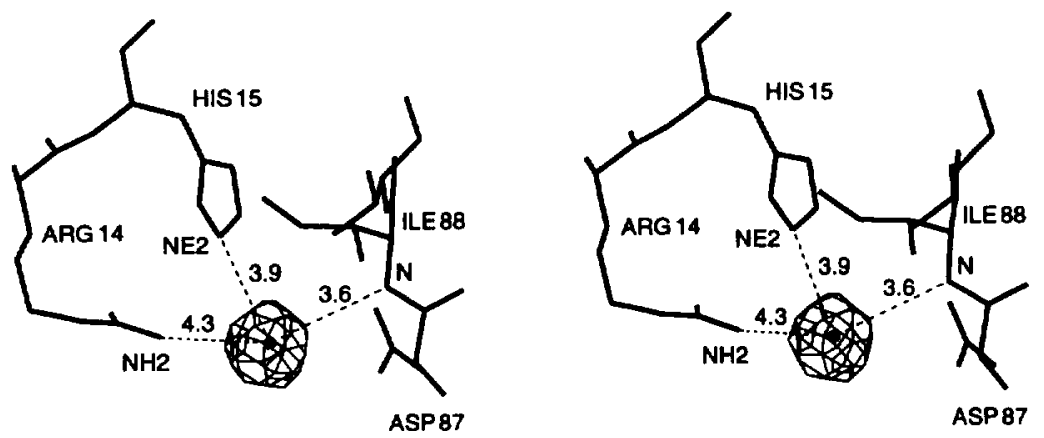

(c)
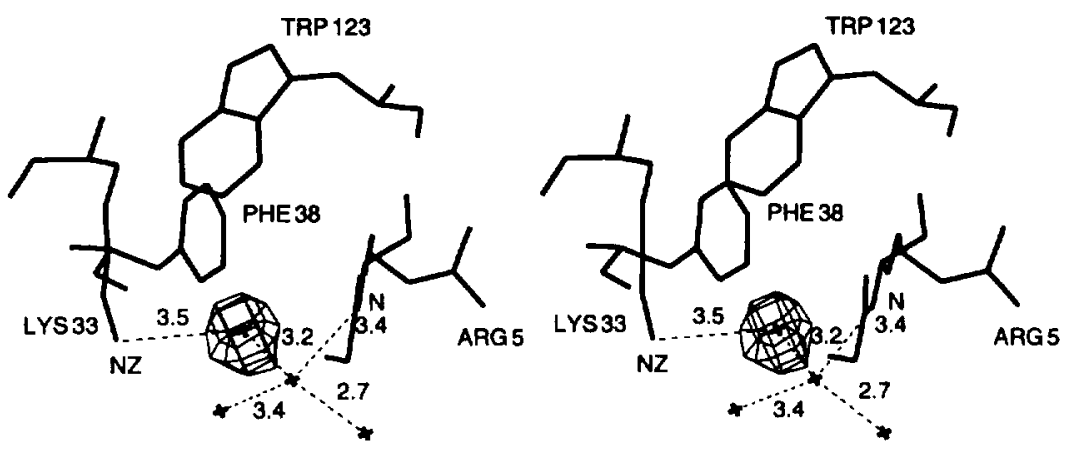

(d)

Fig. 2. Stereo images of the four bromide-ion locations identified by large peaks $(>7 \sigma)$ in the electron-density difference map: (a) the intermolecular Hall site. showing residues from two lysozyme molecules related by 4, symmetry (residues from the second molecule are shown by thin lines), (b) the Hal2 site, (c) the Hal3 site and $(d)$ the Hal4 site. The crosses designate ordered solvent molecules in the vicinity. 
Vancy et al., 1996; Rao \& Sundaralingam, 1996). These locations were then compared with those obtained from this study.

\section{Results}

\subsection{Final models from diffraction data}

A summary of the final refinements is shown in Table 1. Because of the differences in the number of electrons between bromide and chloride (36 and 18 electrons), and assuming that bromide and chloride ions occupy the same positions in the crystal lattice, the bromide positions should be readily identifiable from the $F_{o}(\mathrm{Br})-$ $F_{\text {, }}(\mathrm{Cl})$ map. Four bromide ions were located which showed peak sizes of $7 \sigma$ or greater in the difference Fourier map, along with a relatively weak fifth possible position. One bromide position (designated as Hal1) is at the molecular-crystal interface (Fig. 1) and its electron density is the highest of the four. Hall is closely associated with Tyr23 OH (at a distance of $3.0 \mathrm{~A}$ ) and Asn11.3 ND2 (at a distance of $3.2 \AA$ ) in the symmetryrelated lysozyme (Fig. $2 a$ ). Two water molecules are also close to Hall. Three other bromide ions (Hal2, Hal3 and Hal4) are located in different pockets of the lysozyme molecule in the crystal lattice. Hal2 interacts with the main-chain $\mathrm{N}$ atom of Gly26 and Ser24 OG (Fig. 2b). Hal3 is associated with the basic side chains of Arg14, His 15 and the main-chain $\mathrm{N}$ atom of Ile88 (Fig. 2c). Hal4 is also closely associated with a cationic side chain Lys 33 (the NZ atom at a distance of $3.5 \AA$ ) (Fig. 2d); it also interacts with a bridging water molecule which, in turn, interacts with the main-chain $\mathrm{N}$ atom of Arg5.

The four bromide ions described above are located in the larger of the two lysozyme domains. There is a fifth possible position in the smaller domain (Fig. 3). This site is near Asn74 ND2 and Asn65 ND2 (3.6 and $4.0 \AA$ distant, respectively) and has a relatively small peak size in the difference Fourier map $(\sim 5 \sigma)$. Although it is retained here as a halide ion (Hal5) for reasons discussed in the following sections, there is less certainty that this is a valid anion-binding site. The site is assigned as a water molecule in the refined structure.

\subsection{Other anis n-binding sites}

The site $H: 11$ corresponds to the sole chloride ion binding site re:sorted by Phillips and co-workers (Blake et al., 1967). The carlier study reported a slightly different location (near Arg114 of one molecule and Ser24 in the ot ler) and the reason for this discrepancy is not clcar. However, our location agrees with that from more recent high-resolution studies (Vaney et al., 1996). Six nitrate ion binding sites were reported in monoclinic lysozyme crysials, three sites for each molecule in the dimer asymmetric unit (Rao \& Sundaralingam, 1996). Only four of these sites are unique, as two sites are the same for both molecules in the dimer. Three of these sites correspond to the Hal2, Hal3 and Hal5 sites in tetragonal lysizyme. Two nitrate-binding sites were found from the crystallographic study of low-humidity (closely packed) monoclinic lysozyme crystals (Madhusudan et al., 1993), one of which corresponds to the Hal3 site. Finally, in the binding study with the dyes bromophenol blue and bromophenol red, one binding site was found for each dye (Madhusudan \& Vijayan, 1992). The bromophenol led binding site corresponded to the Hal4 site.

\section{Discussion}

The five anion; found in this study are clearly not the only ones prisent in tetragonal lysozyme crystals. Earlier investi jations using physico-chemical methods found many more anions (Palmer et al., 1948; Carr, 1953; King. 1959). Th e remaining ions are either bound to the crystalline pro ein or free within the solvent channels. When a bounc anion is in contact with the free water present in large channels, it is free to move in the vicinity of a central position (Frey, 1994). Thus, the occupancy of this central position or binding site is likely to be low and will be di ficult to establish by a crystallographic study. This is confirmed by the locations of the five anions determined in this study which are all in small pockets in the crystal, often adjacent to bound water molecules (Fig 2). The absence of free water in these small pockets $\mathrm{r}$ estricts the freedom of movement of the
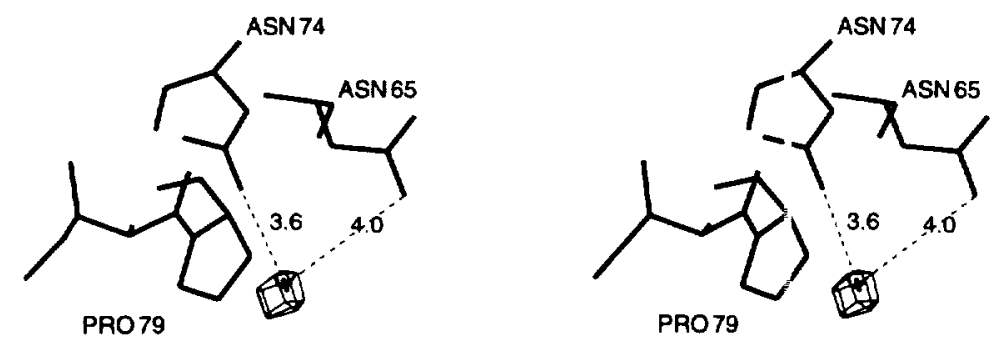

Fig. 3. Stereo image of the Hals site. Given its relatively small peak in the electron-density d fference map ( $\sim 5 \sigma)$, the location of a bromide ion here is not certain and is assigned to a water molccule in the retined structure. However. other evidence suggests that this is indeed a valid anion-hinding site for lysoryme (sec text). 
anion ensuring a high site occupancy (Madhusudan et al., 1993; Frey, 1994), and resulting in high electron densities at these sites.

The anions observed in this study have been designated as halide ions, assuming that both $\mathrm{Cl}^{-}$and $\mathrm{Br}^{-}$ ions occupy these sites. However, the electron-density difference maps only confirm the existence of $\mathrm{Br}^{-}$ions, and $\mathrm{Cl}$ ions may not bind at all these locations. The lower electron density of $\mathrm{Cl}^{-}$and the low overall binding affinity for both $\mathrm{Br}^{-}$and $\mathrm{Cl}^{-}$evident by the high concentrations needed to precipitate lysozyme from solution, make the direct observation of bound $\mathrm{Cl}^{-}$ difficult and its presence can only be inferred from these studies. The presence of both halide ions is assured only at the Hall site, because of earlier observations of a Cl ion at this location. (Blake et al., 1967; Vaney et al., 1996). Two of the halide ions found by this study, Hal3 and Hal4, are associated with basic residues. Hal3 interacts with Arg 14 and His15 (Fig. 2c), making this site a highly basic one and one likely to be occupicd by anions in all crystal structures of this protein. Nitrate ions were also found at this location in the two monoclinic structures of lysozyme (Madhusudan et al., 1993; Rao \& Sundaralingam, 1996).

Hal4 interacts with Lys33, with a bound water molecule associated with the main-chain $\mathrm{N}$ atom of Arg5 (Fig. $2 d$ ) and with the nearby $\operatorname{Arg} 73$ (not shown). This ion has the lowest electron density of the four. suggesting a low site occupancy. Such a low occupancy is most likely to be caused by the relatively large size of this pocket, and it is possible that it binds only the $\mathrm{Br}$ ion and not the smaller $\mathrm{Cl}^{-}$ion. Significantly, this is the only halide ion binding site in the tetragonal form of lysozyme that does not seem to have an equivalent nitrate ion binding site in the monoclinic form.

Hal4 has been identified as the binding site of the dye bromophenol red, which is a much larger molecule (Madhusudan \& Vijayan, 1992). As the dye-bound lysozyme is no longer active against its natural substrate. this site has been suggested as being essential to enzymatic activity by binding to the peptide component of bacterial cell walls. The results here corroborate the importance of this binding site to negatively charged and partially negatively charged groups. The position of Hal4 corresponds very closely with that of the partially negatively charged $\mathrm{O}$ atom in the sulfonate group of bromophenol red (Madhusudan \& Vijayan, 1992). Clearly this site can bind other partially or fully negatively charged groups, such as the $\mathrm{O}$ atoms in the peptide component of bacterial cell walls.

The Hall anion is the only onc located at the interface between lysozyme molecules, where it is close to Arg 114 and associates with partially positively charged atoms on both molecules and bound waters (Fig. 2a). Earlier studies had also indicated that this site was a point of close contact between lysozyme molecules in the tetragonal form (Blake et al., 1967; Imoto et al., 1972;
Nadarajah \& Pusey, 1996) and this accounts for Hal1 having the highest electron density of the five anions located in this study. In fact, the interaction between the molecules at this contact is central to the growth process of tetragonal lysozyme crystals. A chain of these contacts forms strongly bonded helices along the $4_{3}$ crystallographic axes (Nadarajah \& Pusey, 1996). Growth of the crystals proceeds by the formation of aggregates corresponding to the helix in solution, followed by their addition to the crystal face by facetedgrowth mechanisms (Nadarajah et al., 1997). Thus, the Hall anion plays a central role in the formation of tetragonal lysozyme crystals by facilitating the formation of these aggregates in solution (see Fig. 1).

The above discussion suggests that the Hall binding site may be unique to tetragonal lysozyme. The intermolecular contact corresponding to $4_{3}$ symmetry is absent in the monoclinic form and such an intermolecular binding site is not possible. However, one of the $\mathrm{NO}_{3}^{-}$ion-binding sites in monoclinic lysozyme is almost the same as the Hall site, with respect to one molecule only (Rao \& Sundaralingam, 1996). It is near the residues Asn113 and Arg114 (cf. Hall site with respect to the second molecule in Fig. $2 a$ ). Thus, in the same way as the Hal2, Hal3 and Hal5 sites, the Hall can also be considered to have an equivalent anion-binding site in the monoclinic form of lysozyme.

The location of the halide ion Hal2 is somewhat unexpected. Although it does associate with two polar groups, the main-chain $\mathrm{N}$ atom of Gly26 and with the partially charged $\mathrm{H}$ atom in the $\mathrm{OH}$ group of Ser24, there are no positively charged groups in its vicinity in the binding pocket (Fig. $2 b$ ). The importance of this binding site in lysozyme is also suggested by the fact that an $\mathrm{NO}_{3}^{-}$ion binds here in the monoclinic form (Rao \& Sundaralingam, 1996). This may seem surprising, but recent studics of the elcctrostatic fields of proteins in solution suggests that the contributions of polar groups to the field are significant and may even exceed those of charged groups (Warshel \& Áqvist, 1991; Yang et al., 1993). The location of Hal2 near polar, but uncharged, groups confirms their importance to the overall electrostatic field of lysozyme and in particular to anion binding.

Given its low occupancy, some justification is needed for considering the final halide ion binding site Hal5 (Fig. 3) to be occupied by an anion rather than a water molecule. Two reasons can be given. Firstly, there is a sodium ion-binding site at a distance of $8.5 \AA$ (Vaney et al., 1996), which, while rather distant, may increase the chance that an anion site is in the vicinity. Secondly, a nitrate ion was found on this site in the monoclinic form (Rao \& Sundaralingam, 1996), indicating that this may be an anion-binding site in tetragonal lysozyme as well. Nevertheless, even if a halide ion is present at this site in the tetragonal form, it is likely to be only weakly bound to the protein. 
Thus, in the regular monoclinic crystal form of CEWL the four reported nitrate ion binding sites all have equivalent sites occupied by halide ions in the tetragonal form. Among the remaining reported binding sites for anions and dyes, the close-packed monoclinic form binds a nitrate ion at a different, intermolecular site (Madhusudan e' al., 1993). The absence of halide ions at this site can readily be attributed to the different packing of tetragonal crystals. The binding site for bromophenol bluc in tetragonal lysozyme is not at an intermolecular site, but at a large basic pocket near the enzyme cleft. This pocket contains the basic groups Arg21. Lys96 and Lys97 which do not form hydrogen bonds, are accessible to the free solvent and are presumably still protonated. Arg128 is also located in this pocket. As discussed earlier for bromophenol red, the binding of bromophenol blue also inhibits lysozyme activity with its natural substrate (Krishnamoorthy \& Prabhananda, 1982a,b). However, unlike the binding site of bromophenol red which is the same site as Hal4. no halide ions were observed at this site.

The absence of anions on this site may seem surprising given the fact that bromophenol bluc carries two negative charges and that its binding site is a highly basic one. Bromophenol red, on the other hand, is an uncharged but polar molecule with a less basic binding site (Madhusudan \& Vijayan, 1992). The binding site of bromophenol blue is located in a large channel with free water molecules, while $\mathrm{Hal} 4$ is located in a smaller pocket, although as discussed earlier the occupancy of the Hal4 site is also low. This suggests that any anion(s) bound to the bromophenol blue binding site will only have a low occupancy, resulting in low electron densities. Even the much larger bromophenol blue molecule only has an occupancy of 0.30 on this site (Madhusudan \& Vijayan, 1992).

\section{Concluding remarks}

This study shows the effectiveness of using ion-substitution experiments with X-ray crystallography to determine the ion-binding sites of crystalline CEWL. Four likely and one possible halide ion binding sites were found for the tetragonal crystal form. One of these sites corresponded to the intermolccular chloride ion binding site shown by earlier studies. Four of these sites also corresponded to four nitrate ion binding sites found in monoclinic crystals. The remaining binding site was found to correspond to that for the large bromophenol red molec ale in the tetragonal form. These correspondences strongly suggest that the anion-binding sites for crystalline CEWL remain unchanged even when different anions and different crystal forms are employed.

We gratcfully acknowledge the assistance of Dr E. Snell, NAS/NRC resident rescarch associate, NASA/ $M S F C$, in the preparation of this manuscript.

\section{References}

Alderton, G. \& Fevold, H. L. (1946). J. Biol. Chem. 164, I-5. Blake, C. C. F., Mair, G. A., North, A. C. T., Phillips, D. C. \& Sarma, V. ?. (1967). Proc. R. Soc. B, 167, 365-377.

Brünger, A. Г. (1991). Annu. Rev. Phys. Chem. 42, 197-223.

Carr, C. W. (1953). Arch. Biochem. Biophys. 46, 417-423.

Diamond. R. (1974). J. Mol. Biol., 82, 371-391.

Ewing, F. L.. Forsythe. E. L., van der Woerd, M. \& Pusey, M. L. (1996). J. Cryst. Growth. 160, 389-397.

Frey. M. (1904). Acta Cryst. D50, 663-666.

Imoto, T., Johnson, L. N., North, A. C. T., Phillips, D. C. \& Rupley, J. A. (1972). The Enzymes, Vol. 7, edited by P. D. Boyer, pp. 566-889. London: Academic Press.

King. G. S. C. (1959). Acta Cryst 12, 216-219.

Krishnamoor hy, G. \& Prabhananda. B. S. (1982a). Biochim. Biophys. A:ta, 709, 53-57.

Krishnamoor hy, G. \& Prabhananda, B. S. (1982b). Biochim. Biophys. Acta, 709, 234-246.

Madhusudan Kodandapani, R. \& Vijayan, M. (1993). Acta Cryst. D49. 234-245.

Madhusudan \& Vijayan. M. (1992). Protein Eng. 5. 399-404.

Nadarajah, A., Li, M. \& Puscy. M. L. (1997). Acta Cryst. D53. $524-534$.

Nadarajah, A \& Pusey, M. L. (1996). Acta Cryst. D52, 983-996.

Palmer, K. J. Ballantyne, M. \& Galvin, J. A. (1948). I. Am. Chem. Soc. 70, 906-90)6.

Pusey, M. L. iz Munson. S. (1991). J. Cryst. Growth. 113. 385389.

Rao, S. T. \& sundaralingam. M. (1996). Acta Cryst. D52. 170175.

Riès-Kautt. A. M. \& Ducruix, A. F. (1989). J. Biol. Chem. 264, $745-748$.

Steinrauf, L. 3s. (1959). Acta Cryst. 12. 77-79.

Tanford, C. \& Wagner. M. L. (1954). J. Am. Chem. Soc. 76. $3331-3336$.

Vaney, M. C., Maignan, S., Riès-Kautt. M. \& Ducruix, A (1996). Acts Cryst. D52, 505-517.

Warshel, A. \& Aquist, J. (1991). Annu. Rev. Birphys. Biophys. Chem. 20, 257-298.

Yang, A.-S., ( iunner, M. R., Sampogna, R., Sharp. K. A. \& Honig. B. ( 993), Proteins. 15, 252-265. 